\title{
Thermal Kinetic Inductance Detectors Camera: System Level Design, Strategy and Performance Forecast
}

\author{
L. Minutolo, A. Wandui, J. Bock, C. Frez, B. Steinbach, A. Turner, and R. O'Brient
}

\begin{abstract}
The next-generation instruments for millimeterwave astronomy will require large arrays of detectors to surpass current performance [1], [2]. On account of integration challenges, focal planes relying on transition edge sensor (TES) bolometers will not scale gracefully. Thermal Kinetic Inductance Detectors (TKIDs) are highly scalable and have the design flexibility to solve this problem. We present an update on our detector development and systems engineering in preparation for a 2022 TKIDs camera deployment that includes noise performance, optical characterization, and a calibration strategy.
\end{abstract}

Index Terms-KIDs, MKIDs, TKIDs, Observational Cosmology, Readout, RF

\section{INTRODUCTION}

B ICEP array [3] consists of four interchangeable refractive telescopes operating on a common mount [4] situated at the Amundsen-Scott South Pole Station. The BICEP program's goal is to constrain the amplitude of primordial gravitational waves by mapping the polarization of the cosmic microwave background (CMB) [5]. BICEP Array will map the same field on the sky at $30 / 40$ [6], 95, 150, and $220 / 270 \mathrm{GHz}$ to subtract the foreground signals from the B-mode polarization pattern of the CMB. We use TES bolometers to measure frequencies as high as $150 \mathrm{GHz}$ [7] because they are reliable and provide background limited performance. However, the TES bolometers require at least one set of wire-bonds between each detector. Its readout SQUID (superconducting quantum interference devices) is arduous and prone to handling failure at shorter wavelengths with larger numbers of detectors in the focal plane. Implementing and maintaining these connections is a significant point of failure in the telescope. To overcome this scalability challenge, we are retrofitting one of our telescopes to use a focal plane of thermal kinetic inductance detectors (TKIDs) in lieu of TES bolometers. These detectors are RF multiplexed by passive circuits lithographically defined and integrated onto the detector array, alleviating integration

JPL RTD program (2016-19) Strategic support for TKIDs \& readout; President Director Funds, 2018-2020; NASA SAT: started 2020 (JPL); Moore Foundation: started 2019 (Caltech)

(Corresponding author: Lorenzo Minutolo)

L. Minutolo, B. Steinbach, J. Bock and A. Wandui are with the California Institute of Technology, Pasadena, CA 91125, USA (email: minutolo@caltech.edu; awandui@caltech.edu; bsteinba@caltech.edu)

A. Turner, C. Frez and R. O'Brient are with the Jet Propulsion Laboratory, Pasadena, CA 91109, USA (email: anthony.d.turner@jpl.nasa.gov; clifford.f.frez@jpl.nasa.gov; roger.o.brient@jpl.nasa.gov )

Color versions of one or more of the figures in this paper are available online at http://ieeexplore.ieee.org. Digital Object Identifier will be inserted here upon acceptance. concerns. We will deploy a pathfinder TKIDs camera to the South Pole Station in the 2022 Austral Winter to observe at $150 \mathrm{GHz}$, to facilitate comparisons with the prior generation TES bolometer cameras that our group has extensive experience with and data from.

\section{TKIDS TECHNOLOGY}

Thermal kinetic inductance detectors [8], [9] differ from standard KIDs because the inductive element is not designed to absorb photons directly. Instead, an antenna couples the photons to a dissipative gold film on a suspended bolometer island (Fig. 1B), where the inductive element then detects thermalized phonons. The inductor is part of a high Q superconducting resonator, and when phonons on the island break Cooper pairs in the inductor, they shift the resonant frequency. A thermal link, formed by the supporting legs, restores the original island temperature. The legs' thermal conductance (reported as $G_{T}$ in Fig. 2) determines the time constant to restore the detector's idle condition. This detection enjoys multiple advantages over other technologies for our application. In contrast to KIDs, the inductor and absorber in TKIDs are distinct components that can be independently optimized. Moreover, in contrast to TESes, the natural frequency multiplexing minimizes the number of wirebonds to connect to a single RF line per tile [10], [11]. Moreover, the cold electronics are passive - transmission lines and a low noise amplifier (LNA) [12], [13] with none of the active feedback required for readout through SQUIDs [14]. Our test camera will consist of four detector tiles, with 128 antenna-coupled detectors. The focal plane requires one LNA per tile- four total- which is substantially cheaper and easier to operate than the SQUID based multiplexing used for TES bolometer arrays.

\section{Device CALibration}

In addition to the gold antenna termination, we implemented another gold resistor on the island as a calibration heater or calibrator. We can directly bias this resistor with a room temperature current source to precisely mimic the antenna's optical power level $P_{o p t} \sim 5 p W$. Fig. 3 well summarizes the calibration process. By knowing the power dissipated by the calibration heater and measuring the respective resonator properties, we can calibrate the non-linear resonant shift per $p W$ of power in our detector. To limit the number of wires, we connect multiple detectors' calibrators in series with a shared 


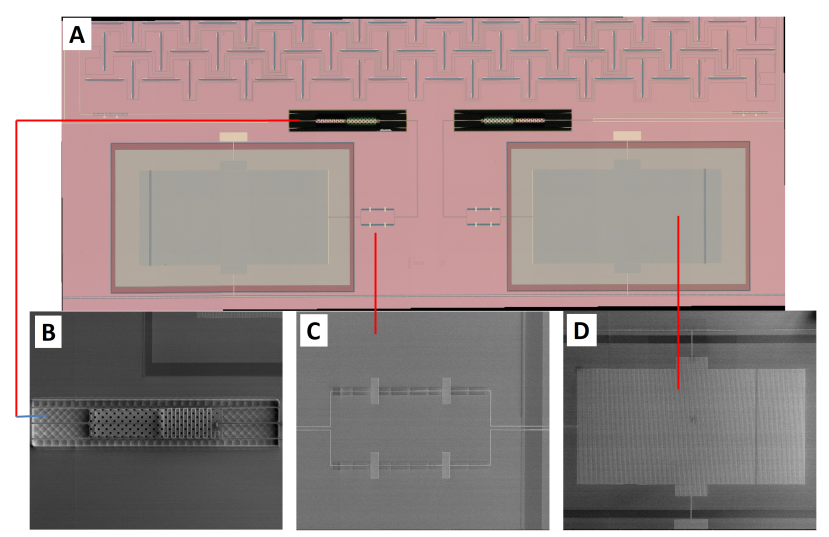

Fig. 1. A) Microscope image of an antenna-coupled TKID with SEMs of the B) TKID island, C) protective RF-choke, and D) resonant and coupling capacitors.

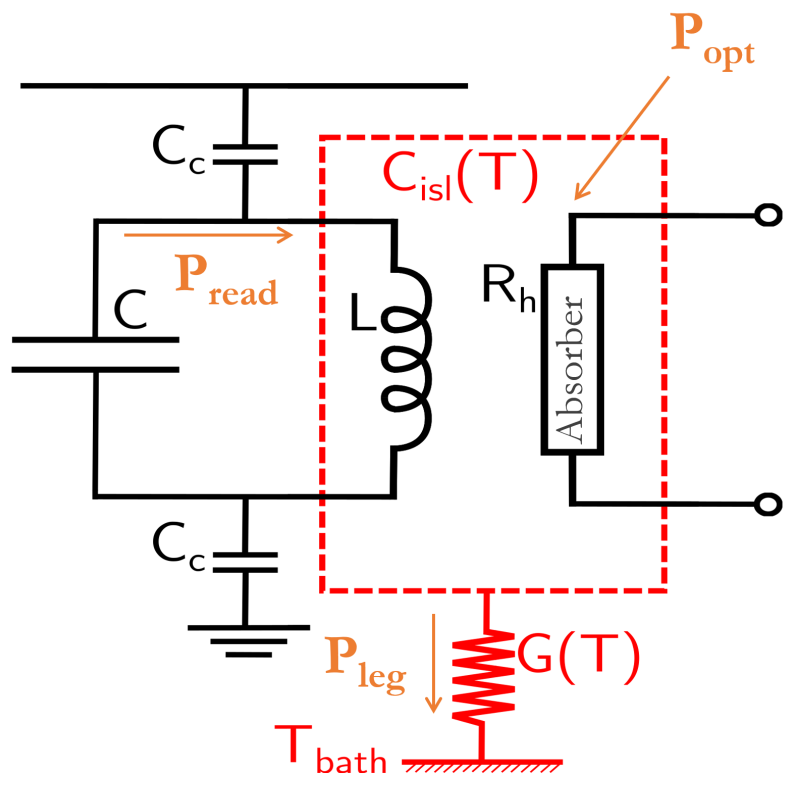

Fig. 2. Electrothermal schematic of a thermal kinetic inductance device (TKID). The incoming photon power or the calibration signal is dissipated on the island (red dotted line) via the gold resistor $R_{h}$. The phonons on the island alter the number of quasiparticle in the inductive element $L$ and are dissipated via the island suspensions or legs, $P_{l e g}$. The thermal conductance of the legs $\mathrm{G}$ can be tuned during the fabrication process [15]. The resonator properties changes are detected using a probe signal with power coming from the room temperature readout electronics, $P_{\text {read }}$

source. The calibrators are used for laboratory characterization and will remain unpowered during observations. We are currently using an open-source GPU based system [10] that takes advantage of commercially available software defined radios (SDR). This system can be easily reconfigured in $\mathrm{C}$ and Python software to allow readout in various customized modes. This readout is especially useful for measurements of room temperature loads that can shift resonators by several linewidths because of our detectors' high responsivity, rendering a traditional fixed-tone comb readout inadequate. Instead, we excite our detectors with a broadband fast chirp [10], that quickly excites the resonators, and Fourier transform their ringdown timestream. While the GPU readout system is

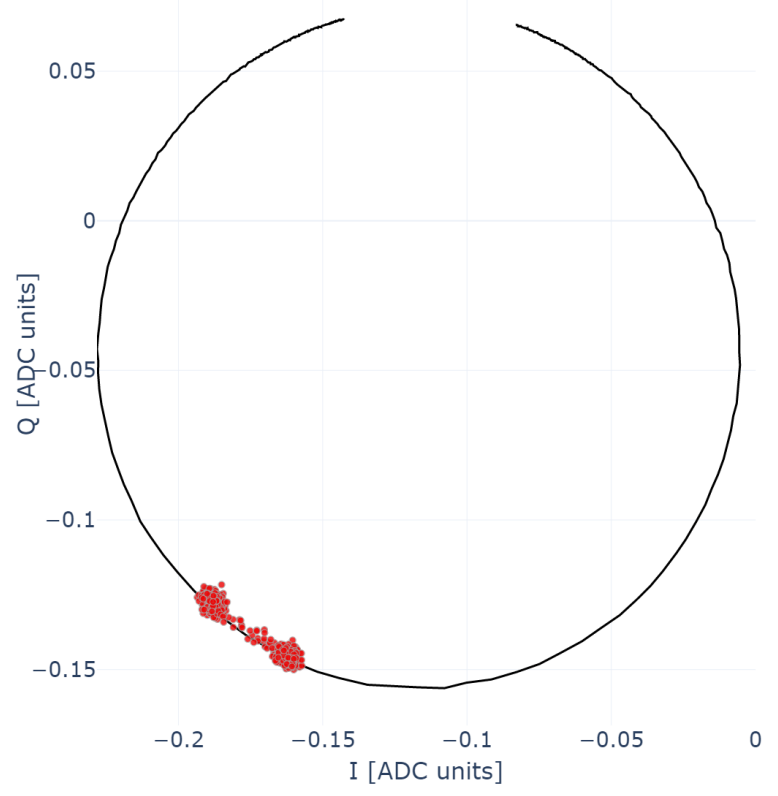

Fig. 3. Calibration of a TKID. A fixed tone acquisition at $469.87 \mathrm{MHz}$ (data points) is plotted over a VNA scan (black line trace) of the same resonator in the IQ plane while the calibration heater is biased with a $0-4 \mathrm{pW}$ square wave. The resonator is first measured with a VNA scan in its unperturbed state; a square wave bias is then applied to the calibration heater while we measure the transmission function at the resonant frequency. This technique allows a direct conversion between noise equivalent frequency (NEF) and noise equivalent power (NEP), like the one shown in Fig. 4

a very effective tool for laboratory characterization, we plan to use different electronics for our field deployment. The software nature of the GPU system, while enabling greater flexibility, reduces the setup's reliability. Stanford developed SMuRF (SLAC Microresonator Radio Frequency) [16] electronics, an FPGA readout platform in which tones are continuously generated and updated in the FPGA at baseband and up/downmixed to feed into or read back from the cryostat (tone tracking). We are currently working toward the integration of SMuRF with TKIDs.

\section{NOISE PERFORMANCE AND STABILITY}

Under the expected loading at the South Pole [9], we understand well the noise spectrum of our TKIDs. The internal dominant noise source changes with optical loading level: if $P_{\text {opt }} \sim[0-5] p W$, then the device behaves as a KID, with internal noise dominated by quasiparticle generation and recombination. However, if $P_{o p t}>5 p W$, phonons in the suspension legs dominate, leading to bolometric behavior. We will operate our focal plane at $300 \mathrm{mK}$ and expect a $6 \mathrm{pW}$ optical loading [17]. While slewing the telescopes across the sky, data in our timestream occupies the $0.1-10 \mathrm{~Hz}$ audio range, and we need to achieve background limited performance in that band. As is evident in Fig. 4, the noise level of most detectors already satisfies this requirement without the need for common-mode noise subtraction techniques. By subtracting common-mode noise from an array of detectors, we can reduce the $1 / f$ knee introduced by the phase instability of the local oscillators 


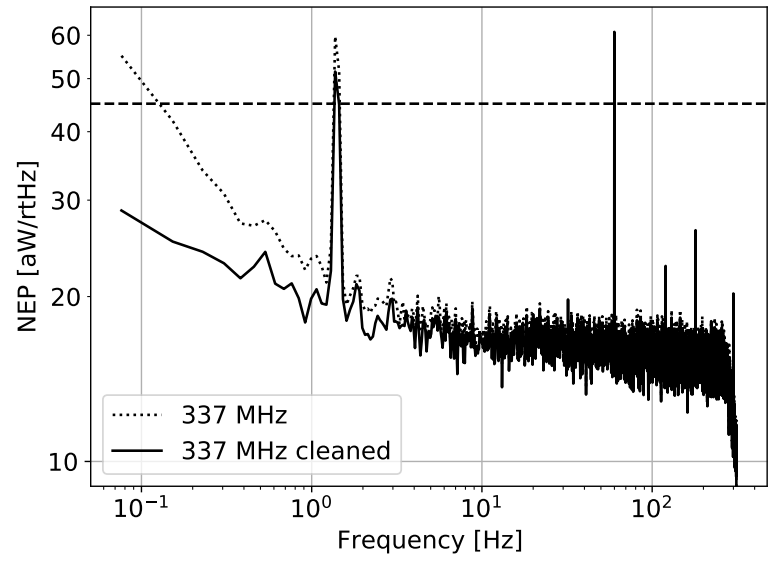

Fig. 4. Calibrated noise spectrum from unbiased, dark TKID expressed in Noise Equivalent Power (NEP). The previous generation of devices we fabricated does not have an antenna and only has the inductor and the calibration heater on the island. We calibrated the devices with the technique described in the Device Calibration section. The dotted horizontal line represents the photon noise we expect from the South Pole sky. The mechanical excitation from our cryostat's pulse tube generates the $1.4 \mathrm{~Hz}$ spike in the spectra. The lowest noise level, labeled as cleaned, is obtained by removing the common mode noise from the $337 \mathrm{MHz}$ detector. The $200 \mathrm{~Hz}$ knee of the spectra reflects the digital filters of the analysis pipeline

(LOs), instability of LNAs power supply, and other roomtemperature electronic noise. Common-mode noise subtraction will naturally occur in our analysis pipeline as we difference orthogonal polarization pairs in each pixel.

\section{OPTICAL PERFORMANCE}

Fig. 5 shows a near field beam map of an antenna coupled device. The near field beam map was measured by moving a hot black body source just above the cryostat optical window. A mechanical chopper with $\mathrm{a} \sim 10 \mathrm{~Hz}$ frequency modulates The black body radiation. The beam profile is not entirely gaussian, and we attribute this partial result to the fact that we are using non-optimized optics in front of our detectors.

We implemented an RF choke, visible in Fig. $1 \mathrm{C}$, between each inductor and capacitor to limit direct stimulation of the TKID that might bypass the integrated antenna and filter. Simulations and prior experience from other KID teams suggested that the resonator circuit could act as a low efficiency antenna and couple out of band photons to the inductor. The detector array under test also included "dark" detectors without antennas to characterize this effect, and they showed a negligible response to thermal loads outside the camera. This result is significant as it represents the first optical signal detected with our antenna-coupled TKIDs (AC-TKIDs) prototypes in a laboratory test environment. The successful light detection and the absence of dark pickup are important milestones towards a TKIDs camera's field deployment.

\section{TESTBED CONFIGURATION}

The testing has been carried on in a Keck-style cryostat, retrofitted to accept RF transmission lines and electronics instead of the time domain multiplexing (TDM) hardware.

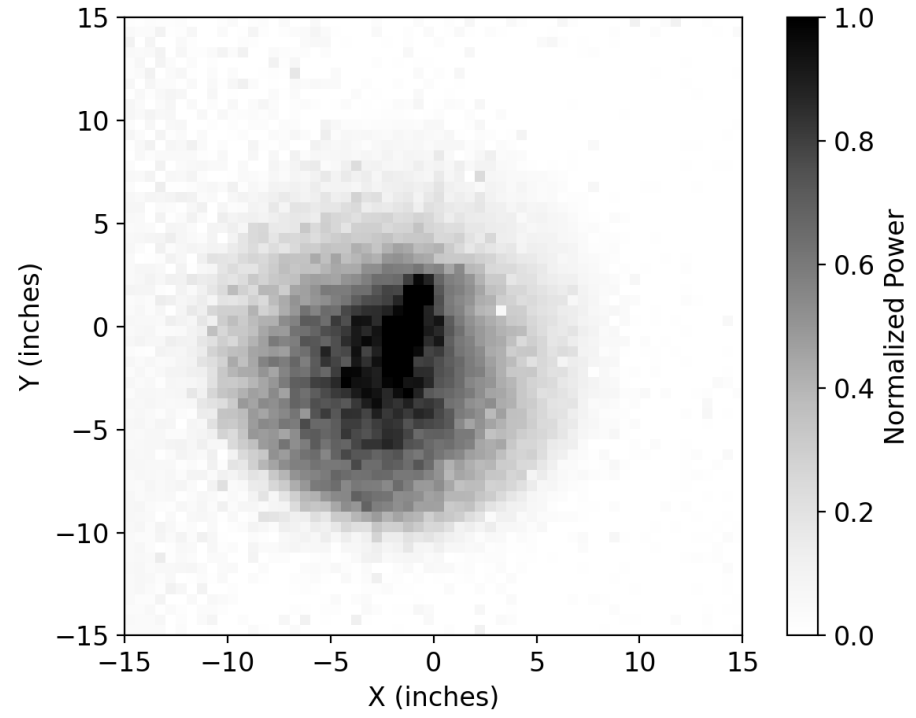

Fig. 5. Near field beam mapping of an antenna-coupled TKID (ACTKID). This particular measure has been realized using the fastchirp readout technique, described in [10], due to the very high responsivity of the resonator.

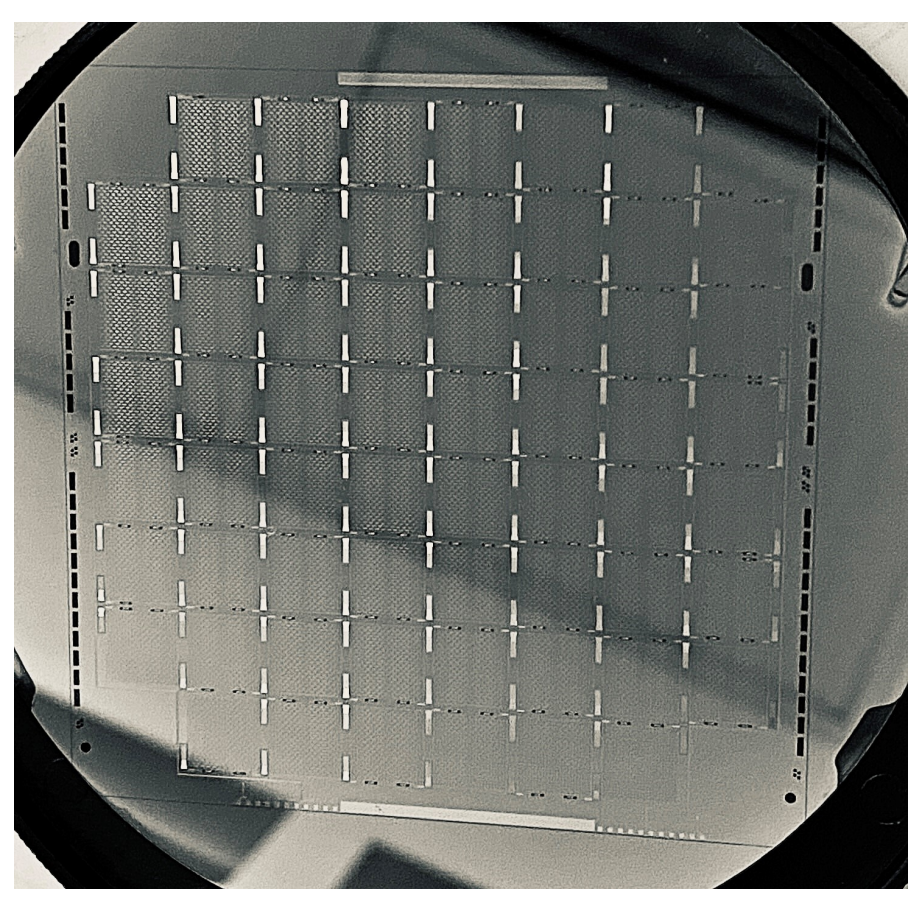

Fig. 6. 128 TKIDs tile during fabrication at JPL. The textured rectangles on the wafer are the antennae of the devices.

The alterations needed to switch between TDM and RF setup, due to the nature of our devices, represent a simplification of the system design. The heat load introduced by the cables is much lower than the TDM configuration as we need to use fewer lines. The space occupied by the cold electronics, mainly at the $4 \mathrm{~K}$ stage, is drastically reduced as we do not need SQUIDs but only four LNAs, which have a significantly smaller footprint. Moreover, the control signal lines for the cold electronics scale down to a single power supply line per amplifier connected to a single low noise, room temperature power supply. This cryostat, called $\mathrm{K} 0$, is compatible with 
the South pole infrastructure and can be directly mounted in BICEP array mount. To speed-up our testing effort, we are implementing new cryostat shells to reduce the open/close time from about a week to a single day. Once the four tile focal plane's testing phase concludes, we will reassemble the original shells to ensure compatibility with South Pole infrastructure.

\section{CONCLUSION}

Our prototype AC-TKIDs arrays have met most of the requirements needed for deployment. We are currently fabricating four AC-TKIDs tiles, with 128 detectors each (a prototype is shown in Fig. 6. Each tile will also feature eight calibration circuits that we will use to calibrate the detectors. We plan to deploy the camera at the South pole for observations during the 2022 Austal Winter. This will map our field at $150 \mathrm{GHz}$ to benchmark the performances against currently deployed TES bolometers. The successful deployment of a $150 \mathrm{GHz}$ TKIDs camera at the South Pole will also advance TKIDs TRL (Technology Readiness Level).

\section{REFERENCES}

[1] H. Hui, P. A. R. Ade, Z. Ahmed, K. D. Alexander, M. Amiri, D. Barkats, S. J. Benton, C. A. Bischoff, J. J. Bock, H. Boenish, R. Bowens-Rubin, R. Bowens-Rubin, I. Buder, E. Bullock, V. Buza J. Connors, J. P. Filippini, S. Fliescher, J. A. Grayson, M. Halpern, S. Harrison, G. C. Hilton, V. V. Hristov, K. D. Irwin, J. Kang, K. S. Karkare, E. Karpel, S. Kefeli, S. A. Kernasovskiy, J. M. Kovac, C. L. Kuo, E. M. Leitch, M. Lueker, K. G. Megerian, V. Monticue, T. Namikawa, C. B. Netterfield, H. T. Nguyen, R. O'Brient, R. W. Ogburn, C. Pryke, C. D. Reintsema, S. Richter, R. Schwarz, C. Sorensen, C. D. Sheehy, Z. K. Staniszewski, B. Steinbach, G. P Teply, K. L. Thompson, J. E. Tolan, C. Tucker, A. D. Turner, A. G. Vieregg, A. Wandui, A. C. Weber, D. V. Wiebe, J. Willmert, W. L. K. $\mathrm{Wu}$, and K. W. Yoon, "BICEP3 focal plane design and detector performance," in Millimeter, Submillimeter, and Far-Infrared Detectors and Instrumentation for Astronomy VIII, ser. Society of Photo-Optical Instrumentation Engineers (SPIE) Conference Series, W. S. Holland and J. Zmuidzinas, Eds., vol. 9914, Jul. 2016, p. 99140T. [Online]. Available: https://arxiv.org/abs/1607.06861

[2] P. A. R. Ade, Z. Ahmed, R. W. Aikin, K. D. Alexander, D. Barkats, S. J. Benton, C. A. Bischoff, J. J. Bock, R. Bowens-Rubin, J. A. Brevik, I. Buder, E. Bullock, V. Buza, J. Connors, J. Cornelison, B. P. Crill, M. Crumrine, M. Dierickx, L. Duband, C. Dvorkin, J. P. Filippini, S. Fliescher, J. Grayson, G. Hall, M. Halpern, S. Harrison, S. R. Hildebrandt, G. C. Hilton, H. Hui, K. D. Irwin, J. Kang, K. S. Karkare, E. Karpel, J. P. Kaufman, B. G. Keating, S. Kefeli, S. A. Kernasovskiy, J. M. Kovac, C. L. Kuo, N. A. Larsen, K. Lau, E. M. Leitch, M. Lueker, K. G. Megerian, L. Moncelsi, T. Namikawa, C. B. Netterfield, H. T. Nguyen, R. O’Brient, R. W. Ogburn, S. Palladino, C. Pryke, B. Racine, S. Richter, A. Schillaci, R. Schwarz, C. D. Sheehy, A. Soliman, T. St. Germaine, Z. K. Staniszewski, B. Steinbach, R. V. Sudiwala, G. P. Teply, K. L. Thompson, J. E. Tolan, C. Tucker, A. D. Turner, C. Umiltà, A. G. Vieregg, A. Wandui, A. C. Weber, D. V. Wiebe, J. Willmert, C. L. Wong, W. L. K. Wu, H. Yang, K. W. Yoon, and C. Zhang, "Constraints on primordial gravitational waves using planck, wmap, and new bicep2/keck observations through the 2015 season," Phys. Rev. Lett., vol. 121, p. 221301, Nov 2018. [Online]. Available: https://link.aps.org/doi/10.1103/PhysRevLett.121.221301

[3] A. Schillaci, P. A. R. Ade, Z. Ahmed, M. Amiri, D. Barkats, R. B. Thakur, C. A. Bischoff, J. J. Bock, H. Boenish, and E. e. a. Bullock, "Design and performance of the first bicep array receiver," Journal of Low Temperature Physics, vol. 199, no. 3-4, p. 976-984, Feb 2020. [Online]. Available: http://dx.doi.org/10.1007/s10909-020-02394-6
[4] M. Crumrine, P. A. R. Ade, Z. Ahmed, R. W. Aikin, K. D. Alexander, D. Barkats, S. J. Benton, C. A. Bischoff, J. J. Bock, R. Bowens-Rubin, J. A. Brevik, I. Buder, E. Bullock, V. Buza, J. Connors, J. Cornelison, B. P. Crill, M. Dierickx, L. Duband, C. Dvorkin, J. P. Filippini, S. Fliescher, J. A. Grayson, G. Hall, M. Halpern, S. A. Harrison, S. R. Hildebrandt, G. C. Hilton, H. Hui, K. D. Irwin, J. H. Kang, K. S. Karkare, E. Karpel, J. P. Kaufman, B. G. Keating, S. Kefeli, S. A. Kernasovskiy, J. M. Kovac, C. L. Kuo, N. A. Larsen, K. Lau, E. M. Leitch, M. V. Lueker, K. G. Megerian, L. Moncelsi, T. Namikawa, C. B. Netterfield, H. T. Nguyen, R. O'Brient, R. W. O. IV, S. Palladino, C. Pryke, B. Racine, S. Richter, R. Schwarz, A. Schillaci, C. D. Sheehy, A. Soliman, T. S. Germaine, Z. K. Staniszewski, B. Steinbach, R. V. Sudiwala, G. P. Teply, K. L. Thompson, J. E. Tolan, C. E. Tucker, A. D. Turner, C. Umiltà, A. G. Vieregg, A. Wandui, A. C. Weber, D. V. Wiebe, J. Willmert, C. L. Wong, W. L. K. Wu, E. Yang, K. W. Yoon, and C. Zhang, "BICEP Array cryostat and mount design," in Millimeter, Submillimeter, and Far-Infrared Detectors and Instrumentation for Astronomy IX, J. Zmuidzinas and J.-R. Gao, Eds., vol. 10708, International Society for Optics and Photonics. SPIE, 2018, pp. 303 - 312. [Online]. Available: https://doi.org/10.1117/12.2312829

[5] K. N. Abazajian, P. Adshead, Z. Ahmed, S. W. Allen, D. Alonso, K. S. Arnold, C. Baccigalupi, J. G. Bartlett, N. Battaglia, B. A. Benson, C. A. Bischoff, J. Borrill, V. Buza, E. Calabrese, R. Caldwell, J. E. Carlstrom, C. L. Chang, T. M. Crawford, F.-Y. Cyr-Racine, F. D. Bernardis, T. de Haan, S. di Serego Alighieri, J. Dunkley, C. Dvorkin, J. Errard, G. Fabbian, S. Feeney, S. Ferraro, J. P. Filippini, R. Flauger, G. M. Fuller, V. Gluscevic, D. Green, D. Grin, E. Grohs, J. W. Henning, J. C. Hill, R. Hlozek, G. Holder, W. Holzapfel, W. Hu, K. M. Huffenberger, R. Keskitalo, L. Knox, A. Kosowsky, J. Kovac, E. D. Kovetz, C.-L. Kuo, A. Kusaka, M. L. Jeune, A. T. Lee, M. Lilley, M. Loverde, M. S. Madhavacheril, A. Mantz, D. J. E. Marsh, J. McMahon, P. D. Meerburg, J. Meyers, A. D. Miller, J. B. Munoz, H. N. Nguyen, M. D. Niemack, M. Peloso, J. Peloton, L. Pogosian, C. Pryke, M. Raveri, C. L. Reichardt, G. Rocha, A. Rotti, E. Schaan, M. M. Schmittfull, D. Scott, N. Sehgal, S. Shandera, B. D. Sherwin, T. L. Smith, L. Sorbo, G. D. Starkman, K. T. Story, A. van Engelen, J. D. Vieira, S. Watson, N. Whitehorn, and W. L. K. Wu, "Cmb-s4 science book, first edition," 2016. [Online]. Available: https://arxiv.org/abs/1610.02743

[6] M. J. Myers, W. Holzapfel, A. T. Lee, R. O’Brient, P. L. Richards, H. T. Tran, P. Ade, G. Engargiola, A. Smith, and H. Spieler, "An antenna-coupled bolometer with an integrated microstrip bandpass filter," Applied Physics Letters, vol. 86, no. 11, p. 114103, 2005. [Online]. Available: https://doi.org/10.1063/1.1879115

[7] C. Zhang, P. A. R. Ade, Z. Ahmed, M. Amiri, D. Barkats, R. B. Thakur, C. A. Bischoff, J. J. Bock, H. Boenish, E. Bullock, and et al., "Characterizing the sensitivity of 40 ghz tes bolometers for bicep array," Journal of Low Temperature Physics, vol. 199, no. 3-4, p. 968-975, Feb 2020. [Online]. Available: http://dx.doi.org/10.1007/s10909-02002411-8

[8] B. A. Steinbach, J. J. Bock, H. T. Nguyen, R. C. O'Brient, and A. D. Turner, "Thermal kinetic inductance detectors for groundbased millimeter-wave cosmology," Journal of Low Temperature Physics, vol. 193, no. 3-4, p. 88-95, Jul 2018. [Online]. Available: http://dx.doi.org/10.1007/s10909-018-2016-y

[9] A. Wandui, J. J. Bock, C. Frez, M. Hollister, L. Minutolo, H. Nguyen, B. Steinbach, A. Turner, J. Zmuidzinas, and R. O'Brient, "Thermal kinetic inductance detectors for millimeter-wave detection," Journal of Applied Physics, vol. 128, no. 4, p. 044508, Jul 2020. [Online]. Available: http://dx.doi.org/10.1063/5.0002413

[10] L. Minutolo, B. Steinbach, A. Wandui, and R. O'Brient, "A flexible gpu-accelerated radio-frequency readout for superconducting detectors," IEEE Transactions on Applied Superconductivity, vol. 29, no. 5, pp. 1-5, 2019. [Online]. Available: https://arxiv.org/abs/1812.02200

[11] S. McHugh, B. A. Mazin, B. Serfass, S. Meeker, K. O’Brien, R. Duan, R. Raffanti, and D. Werthimer, "A readout for large arrays of microwave kinetic inductance detectors," Review of Scientific Instruments, vol. 83, no. 4, p. 044702, Apr 2012. [Online]. Available: http://dx.doi.org/10.1063/1.3700812

[12] S. Weinreb, J. C. Bardin, and H. Mani, "Design of cryogenic sige low-noise amplifiers," IEEE Transactions on Microwave Theory and Techniques, vol. 55, no. 11, pp. 2306-2312, 2007. [Online]. Available: https://ieeexplore.ieee.org/document/4359086

[13] Cosmic Microwave Technology Inc., "Citlf2 cryogenic SiGe low noise amplifier." [Online]. Available: https://www.cosmicmicrowavetechnology.com/citlf2

[14] J. Clarke and A. I. Braginski, "The squid handbook," John 
Wiley \& Sons, Ltd, pp. i-xvi, 2004. [Online]. Available: https://onlinelibrary.wiley.com/doi/abs/10.1002/3527603646.fmatter

[15] E. A. Williams, S. Withington, C. N. Thomas, D. J. Goldie, and D. Osman, "Superconducting transition edge sensors with phononic thermal isolation," Journal of Applied Physics, vol. 124, no. 14, p. 144501, 2018. [Online]. Available: https://doi.org/10.1063/1.5041348

[16] S. A. Kernasovskiy, S. E. Kuenstner, E. Karpel, Z. Ahmed, D. D. Van Winkle, S. Smith, J. Dusatko, J. C. Frisch, S. Chaudhuri, H. M. Cho, and et al., "Slac microresonator radio frequency (smurf) electronics for read out of frequency-division-multiplexed cryogenic sensors," Journal of Low Temperature Physics, vol. 193, no. 3-4, p. 570-577, May 2018. [Online]. Available: http://dx.doi.org/10.1007/s10909-018-1981-5

[17] P. L. Richards, "Bolometers for infrared and millimeter waves," Journal of Applied Physics, vol. 76, no. 1, pp. 1-24, 1994. [Online]. Available: https://doi.org/10.1063/1.357128 